\title{
RICHESSE EN VITAMINES DU LAIT LIQUIDE ET DU LAIT SEC
}

\author{
par G. C. SUPPLEE, Odessa D. DOW et J. W. NELSON
}

du Laboratoire de Recherches de The Dry Milk Company, New-York.

La plus grande partie des renseignements expérimentaux, relatifs aux causes des variations de la richesse en vitamines des produits de la laiterie, a été obtenue avec le lait naturel liquide, et ce n'est qu'occasionnellement qu'on trouve dans la littérature des études relatives à des produits lactés fabriqués. Etant donné que le lait sec est maintenant très apprécié, aussi bien au point de vue économique qu'au point de vue diététique, et en raison de ce que ses méthodes de préparation comportent des phases différentes de celles de presque tous les autres produits lactés, des études plus systématiques lui ont été consacrées que connaissent moins les autres produits lactés manufacturés. Toutefois, à notre connaissance, on n'a pas encore publié de renseignements montrant la richesse comparative des 3 vitamines communes " $\mathrm{A} \|_{2}$ ( $B$ » et " $C$ » dans les mêmes échantillons de lait avant et après dessiccation. Cet article a pour but de donner les résultats d'une telle étude. Pour faciliter une plus large interprétation des résultats, dont les valeurs comparatives pourront être appliquées à des produits commerciaux standardisés, aucune modification expérimentale n'a été apportée aux conditions de traitement du lait liquide avant dessiccation ou pendant la dessiccation elle-même.

La période de juin à octobre a été choisie pour faire ces études comparatives. Conformément aux méthodes habituellement employées dans la recherche des vitamines, des cochons d'Inde et des rats blancs élevés, à l'aide de rations expérimentales éprouvées, puis standardisées, dans nos propres stations d'élevage, furent utilisés. Le lait pour les essais, liquide ou sec, était obtenu journellement d'une fabrique commerciale de lait sec. Les échantillons de lait liquide furent prélevés dans les grandes cuves servant à l'alimentation des cylindres dessiccateurs Just. En même temps que l'échantillon de lait liquide était prélevé, on prélevait un échantillon de lait sec provenant du même lot de lait. Antérieurement à la dessiccation proprement dite, le lait liquide était soumis au traitement habituel usité dans cette fabrique : à aucun moment, avant la dessiccation, le lait n'était chauffé. La méthode habituelle de la fabrique est de refroidir le lait à $45^{\circ} \mathrm{F}$. $\left(7^{\circ}, 22 \mathrm{C}\right.$.) dès réception, et de le dessécher immédiatement. On n'employa ni le carbonate de soude, ni aucun autre neutralisant. Etant donné que le lait liquide et le lait sec reconstitué en lait liquide étaient donnés immédiatement aux animaux en expérience, il est évident que les facteurs qui pouvaient rendre les résultats non concluants étaient éliminés. 
COMPARAISON DE LA RICHESSE EN VITAMINE "A, DE LAIT LIQUIDE ET DE LAIT (DRYCO)

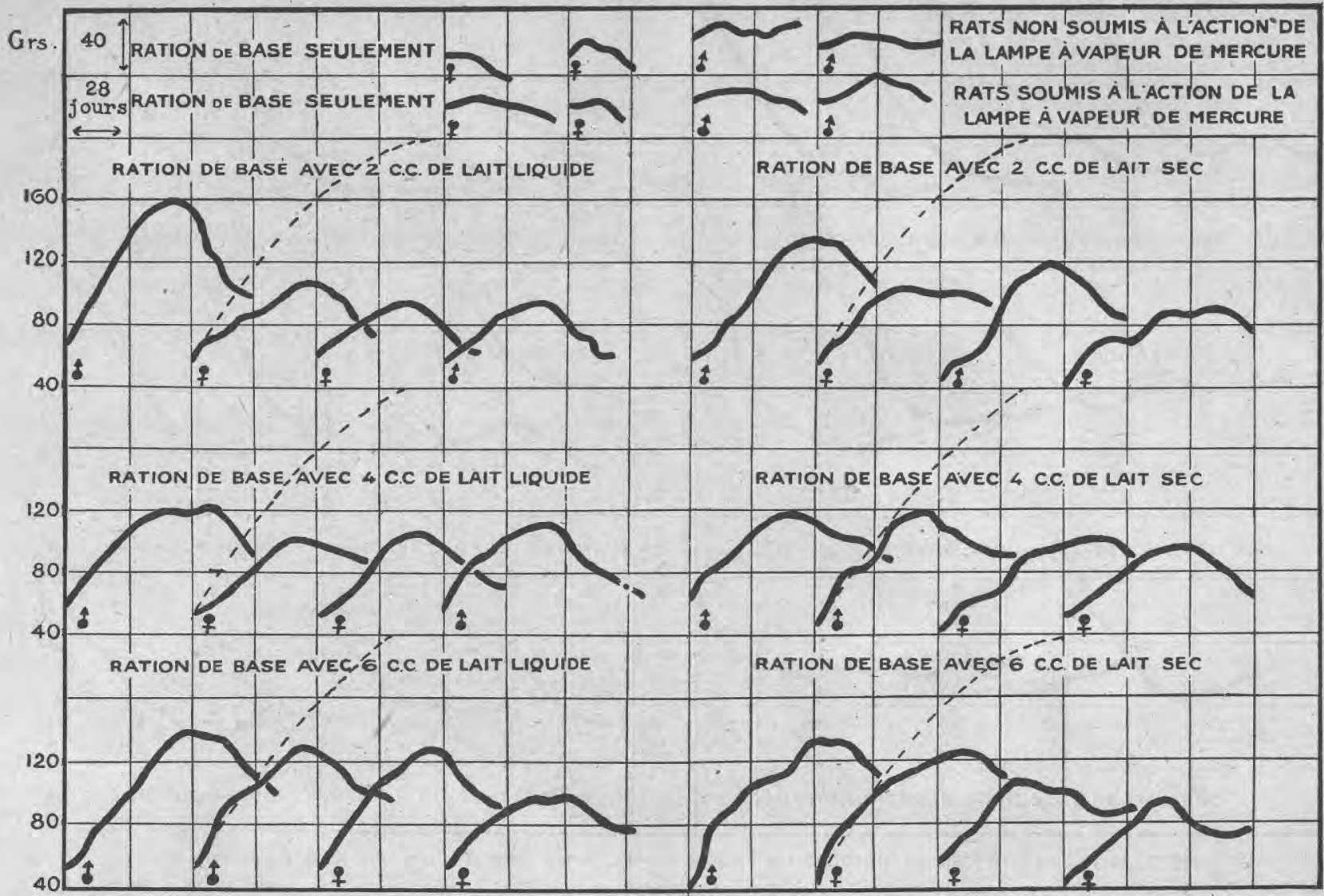


TABLEAU "I

COMPARAISON DE LA RICHESSE EN VITAMINE "A.,DE LAIT LIQUIDE ET DE LAIT SEC (DRYCO)

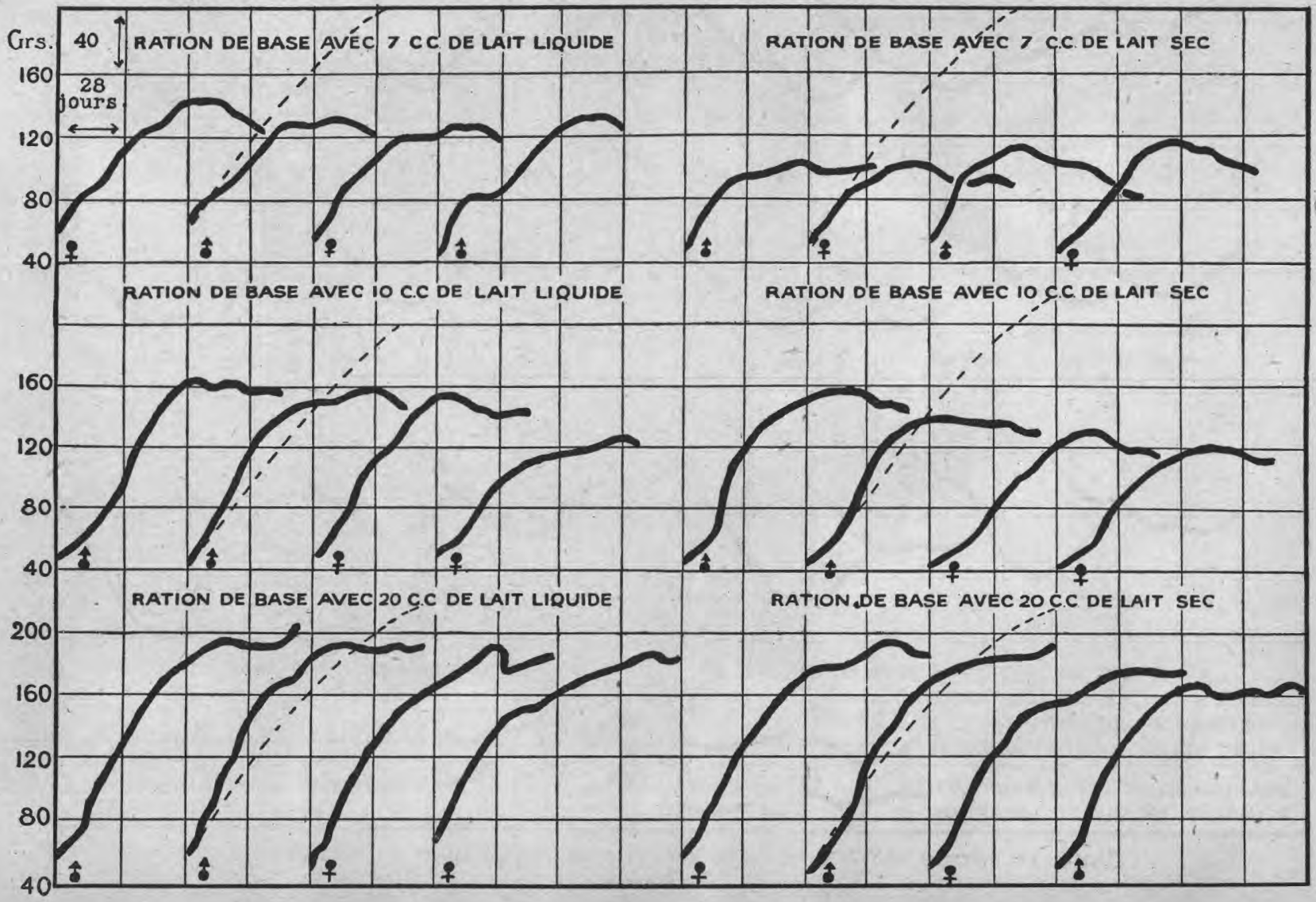




\section{Vitamine A}

En raison des divergences d'opinion au sujet de la quantité de lait nécessaire pour fournir suffisamment en vitamine A une croissance normale, et du fait que nous employions un lait contenant une pròportion réduite de matière grasse (marque Dryco contenant $12 \%$ dans le lait sec et environ $1,2 \%$ dans le lait liquide), nous fûmes obligés de recourir à une grande variété de quantités pour avoir des renseignements convenables, en ce qui concerne l'existence ou la nonexistence d'un effet destructif dû à la dessiccation.

Les essais d'alimentation eurent lieu avec des rats blancs âgés de 25 à 28 jours, conformément aux règles établies, et tous les facteurs nécessaires à la croissance, autres que la vitamine $\mathrm{A}$, furent fournis. La ration de base consistait en:18 parties de caséine exempte de vitamine ; mélange de sels no 40 [1] : 4 parties; levure sèche : 6 parties ; agar-agar en poudre: 2 parties; et dextrine : 70 parties. La nécessité de fournir une quantité convenable de prineipe anti-rachitique, quand on emploie la méthode de eroissance pour la détermination de la vitamine A, a été démontrée par Steenbock, Nelson et BLACK [2]. C'est pourquoi ce facteur fut fourni en exposant les animaux aux émanations d'une lampe en quartz à vapeur de mercure à une distance de 26 pouces $(=0$ mètre 66 ) pendant 8 minutes chaque jour. Pour empêcher les animaux à l'essai de consommer leurs excréments, des écrans en fil de fer ( 3 mailles par pouce, soit 3 mailles par $0 \mathrm{~m}, 0254$ ) furent placés sur le fond des cages. Le lait à l'essai, liquide ou reeonstitué, fut donné en quantités variant de 2 à 20 cc. par jour comme seule source de vitamine $\mathrm{A}$. Les animaux étaient pesés chaque semaine et les courbes du croissance tracées comme l'indiquent les tableaux I et II.

En comparant les courbes de croissance des animaux recevant de petites quantités de lait et celles des animaux recevant seulement la ration de base, ou la ration de base et le facteur antirachitique fourni par la lampe à vapeur de mercure, on constate l'effet puissant de petites quantités de vitamine A fournie par de très petites quantités de lait. Les résultats montrent que les toutes petites quantités furent trop faibles pour fournir une proportion suffisante de ce facteur. La croissance normale n'eut lieu que lorsque des quantités de 20 cc. du produit liquide ou sec furent données et, même avec cette quantité, on constate un développement retardé juste avant l'âge adulte.

$\mathrm{Du}$ reste, comme le principal objet de ces expériences était plutôt de déterminer l'effet de la dessiccation sur la vitamine A que de déterminer la proportion de lait nécessaire pour fournir une quantité suffisante de ce facteur, les résultats montrent clairement qu'il n'y a pas de différence mesurable dans la richesse en cette vitamine entre le lait sec obtenu par le procédé Just et le lait liquide dont il provient. 


\section{Vitamine B}

Les échantillons de lait, liquide ou sec, employés dans les expériences faites en vue de déterminer la richesse relative en vitamine $B$ de ces produits, furent obtenus d'une façon identique à celle décrite pour les études de la vitamine A. Toutefois, dans ces séries, des essais d'alimentation préliminaires eurent lieu pour déterminer la valeur de eroissance qu'il était possible d'espérer quand des quantités croissantes de lait sec reconstitué en lait liquide étaient données sans comparaisons parallèles avec le produit liquide naturel. La technique des essais d'alimentation fut seulement modifiée dans la limite nécessaire pour répondre aux conditions imposées pour la détermination de la vitamine B. La ration de base se composait de: caséine exempte de vitamine B : 18 parties; mélange de sels no 40 [1] : 4 parties ; graisse de beurre : 5 parties ; agar-agar en poudre : 2 parties ; et dextrine : 71 parties. Les animaux étaient placés sur des écrans en fil de fer pour empêcher la consommation des excreta, disposition qui est obligatoire pour une interprétation convenable des résultats des études sur la vitamine B, car comme l'ont prouvé STEENBock et ses collaborateurs [3], les besoins en vitamine B sont diminués de moitié quand les animaux à l'essai ont leurs excreta à leur disposition. Le facteur antirachitique ne fut pas fourni par de faibles radiations ultra-violettes, car il fut supposé que les 5 pour cent de graisse de beurre dans la ration de base fournissaient une quantité suffisante de ce facteur.

Dans les séries préliminaires, où seul du lait sec reconstitué fut employé, des quantités variant de 2 à $10 \mathrm{cc}$. par jour furent données comme unique source de vitamine B. Les courbes de croissance sont montrées dans le tableau III, sur lequel on notera qu'il y a une régularité marquée dans les augmentations de la croissance lorsque les quantités de lait et de son facteur alimentaire augmentent. Toutefois, même la plus grande quantité $(10 \mathrm{cc}$.) n'est pas suffisante pour permettre un taux normal de croissance jusqu'à l'âge adulte.

Dans le tableau IV, on voit les résultats comparatifs obtenus avec le lait liquide et le lait sec reconstitué quand ces produits sont donnés au taux de 20, 25 et $30 \mathrm{cc}$. par jour. L'examen de ces courbes de croissance montre de suite qu'il n'y a pas de diminution de richesse en vitamine B dans le lait séché par le procédé Just. Les résultats confirment aussi les observations antérieures ayant prouvé que les besoins en vitamine B augmentent graduellement quand l'animal en croissance approche de l'âge adulte, et que les quantités suffisantes pour maintenir un taux normal de croissance pendant les premières périodes de la vie, ne sont pas forcément suffisantes pour le développement normal pendant la période précédant immédiatement l'âge adulte. 
TABLEAU III

RICHESSE EN VITAMINE B DE QUANTITÉS VARIABLES DE LAIT SEC (DRYCO)

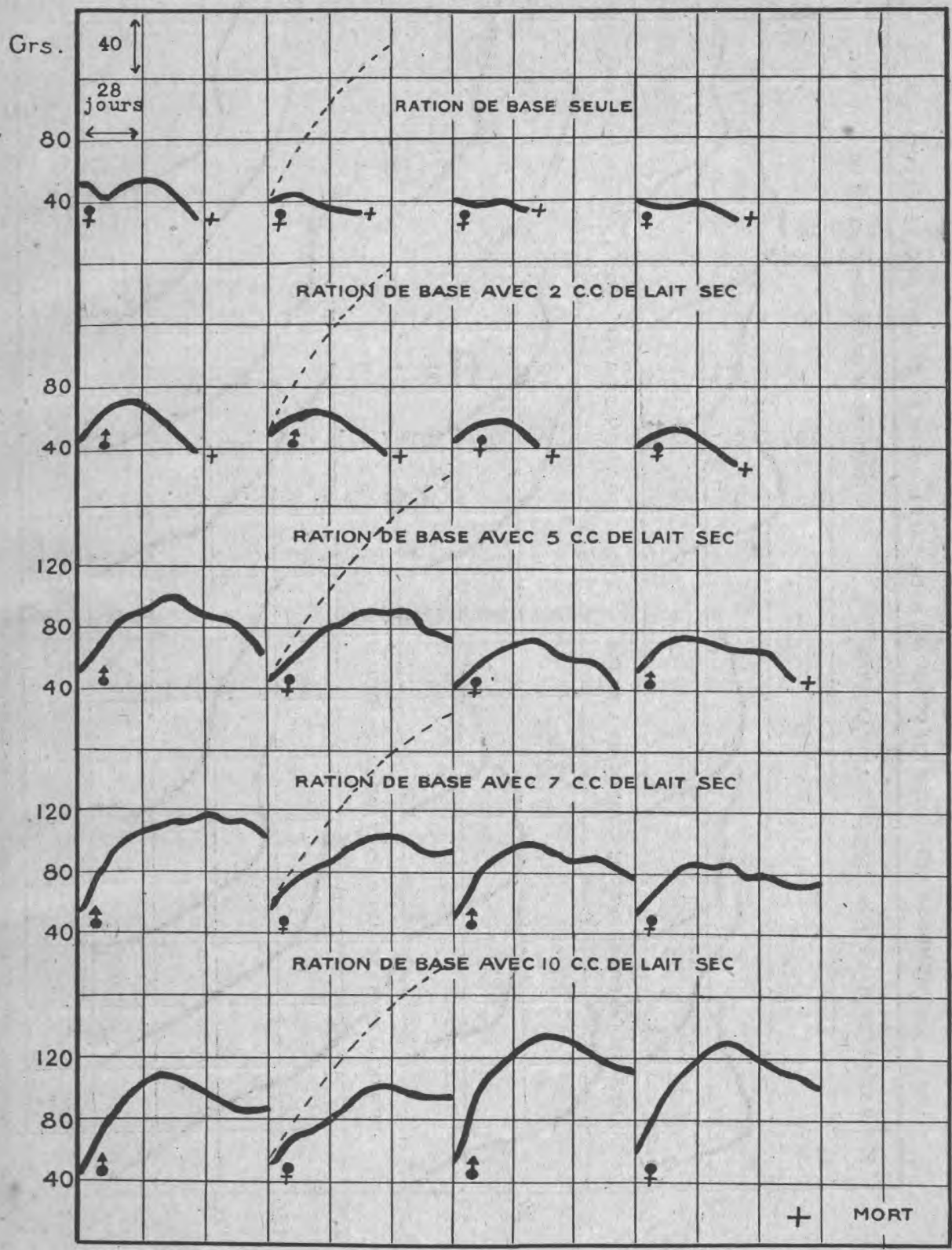


TABLEAU IV

RICHESSE COMPARĖE EN VITAMINE B DE LAIT LIQUIDE ET DE LAIT SEC (DRYCO)

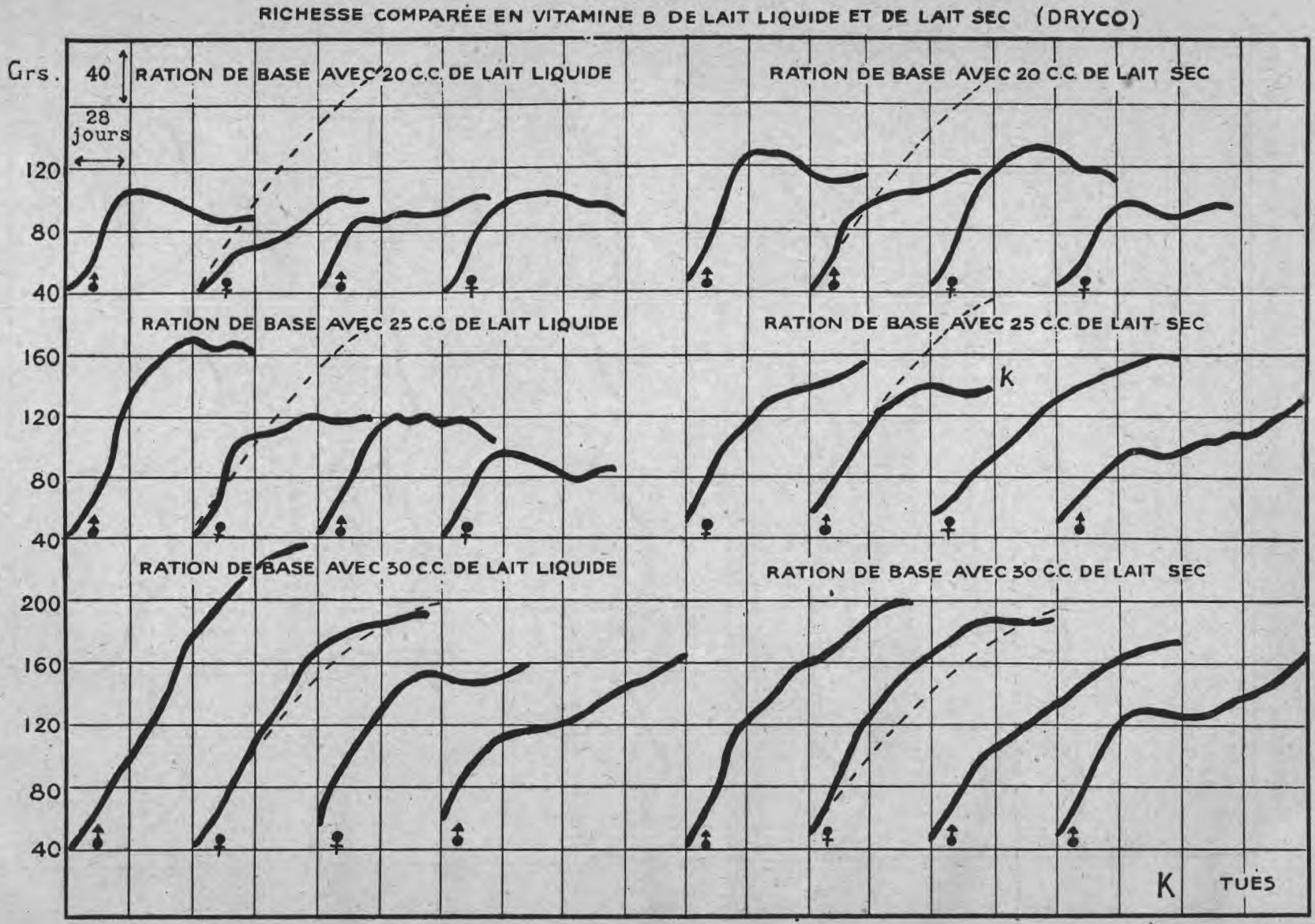


Actuellement, le rôle que la vitamine B joue dans divers phénomènes métaboliques est inconnu. On a cependant observé [4] qu'une insuffisance de ce facteur cause une perte d'appétit qui tend à empêcher la consommation d'une quantité suffisante d'aliments pour parer au déficit. Les chiffres du tableau A ci-dessous illustrent ces rapports. La consommation d'aliment par semaine est comparée avec l'augmentation moyenne de poids par semaine pour les animaux dont les courbes de croissance sont montrées dans les tableaux III et IV: On notera que la ration de base moyenne consommée varie de $24,6 \mathrm{gr}$. ̀̀ $55,8 \mathrm{gr}$. par semaine, car le lait augmente de 0 à 30 cc. par jour. Le gain moyen par semaine pour les animaux ne recevant pas de lait fut de $1 \mathrm{gr} .61$, tandis que la moyenne pour ceux recevant $30 \mathrm{cc}$. de lait, fut de $9 \mathrm{gr} .52$. A noter le fait très particulier que moins de nourriture fut consommée, et que le gain moyen par semaine fut moindre avec le lait liquide qu'avec le lait sec reconstitué donné en quantités semblables. Des observations identiques ont été faites à l'occasion d'autres recherches entı eprises dans ce laboratoire, mais jusqu'à présent, il a été impossible. de donner une explication satisfaisante de cette différence.

\section{TABIEAU A}

Consommation de nourriture et gain moyen en poids par semaine sous l'influence de quantités variables de lait.

\begin{tabular}{|c|c|c|c|c|c|c|}
\hline \multirow{2}{*}{$\begin{array}{l}\text { Nature de } \\
\text { lait donué }\end{array}$} & \multirow{2}{*}{$\begin{array}{c}\text { Quantité } \\
\text { par } \\
\text { jour en } \\
\text { cemes }\end{array}$} & \multirow{2}{*}{$\begin{array}{l}\text { Nombre } \\
\text { de } \\
\text { semaines } \\
\text { de l' lixié- } \\
\text { rielce }\end{array}$} & \multicolumn{2}{|c|}{$\begin{array}{l}\text { Quantité moyenne } \\
\text { heluomadaire d'aliments } \\
\text { consomn és } \\
\text { (fn grammes) }\end{array}$} & \multirow{2}{*}{ Total } & \multirow{2}{*}{$\begin{array}{c}\text { Gain } \\
\text { moyen par } \\
\text { semaine } \\
\text { (en grammes) }\end{array}$} \\
\hline & & & $\begin{array}{c}\text { ration de } \\
\text { base }\end{array}$ & $\begin{array}{c}\text { lait en } \\
\text { extrait sec }\end{array}$ & & \\
\hline Aucun - & Aucun & 6,25 & 24,6 & Aucun & 24,6 & 1,61 \\
\hline Dryco & 2 & 6,50 & 30,1 & 1,4 & 31,5 & 2,31 \\
\hline Dryco & 5 & 10,50 & 32,1 & 3,5 & 35,6 & 3,74 \\
\hline Dryco & 7 & 12,00 & 34,9 & 4,9 & 39,8 & 4,42 \\
\hline Dryco & 10 & 13,00 & 37,5 & 7,0 & 44,5 & 5,08 \\
\hline Dryeo & 20 & 11,50 & 37,5 & 14,0 & 51,5 & 6,06 \\
\hline Liquide & 20 & 11,50 & 30,8 & 14,0 & 44,8 & 4,82 \\
\hline Dryeo & 25 & 14,75 & 45,7 & 17,5 & 63,2 & 6,63 \\
\hline Liquide & 25 & 11,50 & 35,7 & 17,5 & 53,2 & 6,37 \\
\hline Dryeo & 30 & 14,50 & 55,8 & 21,0 & 76,8 & 9,52 \\
\hline Liquide & 30 & 14,50 & 54,6 & 21,0 & 75,6 & 9.13 \\
\hline
\end{tabular}




\section{Vitamine C}

Les résultats des essais qui ont été publiés relativement à la richesse en vitamine $\mathrm{C}$ du lait sec sont quelque peu contradictoires, surtout en ce qui concerne le degré de richesse de ce facteur dans du lait sec préparé par diverses méthodes. Cependant, le plus grand nombre des résultats publiés indique qu'il y a peu ou pas de destruction de ce facteur dans le lait sec obtenu par le procédé des cylindres $(5,6,7,8$ et 9). A notre connaissance, il n'a pas été rendu compte d'essais dans lesquels le même lait a été étudié au point de vue de sa valeur antiscorbutique avant et après dessiccation. Nous avons fait ces comparaisons en employant la même méthode pour obtenir les échantillons que dans les études précédentes sur la vitamine A et la vitamine B.

Pour ces essais, un choix de cochons d'Inde jeunes et vigoureux, de poids et d'âge uniformes, fut fait dans notre colonie d'élevage qui reçoit une ration standardisée ne permettant pas l'emmagasinement d'un excédent abondant du facteur anti-scorbutique dans les organes de l'animal [9]. La ration scorbutique de base utilisée pour les essais consistait en : avoine roulée : 99 parties ; chlorure de sodium : 1 partie ; et foin passé à l'autoclave pendant 45 minutes à 15 livres ( $1 \mathrm{~kg} .05)$ de pression à volonté. Le lait liquide et le lait sec reconstitué furent donnés en quantités journalières de $40,60,70$ et 80 ec. Toute quantité non consommée de la ration journalière était administrée à la pipette. Les faibles quantités furent employées pour voir si le temps qui s'écoule avant le début du seorbut serait suffisant pour déterminer s'il y avait eu une légère détérioration de la vitamine pendant la dessiccation. Quelques difficultés furent éprouvées par suite de désordres digestifs affectant quelques-uns des animaux recevant les plus fortes quantités. Cet incident est indiqué dans la plupart des courbes de croissance des tableaux V, VI, VII et VIII.

Le tableau $V$ montre les courbes de croissance obtenues quand des quantités de $40 \mathrm{cc}$. de lait liquide et de lait sec furent données. On notera, dans quelques cas, que les courbes de croissance sont quelque peu meilleures dans le cas du lait liquide que dans celui du lait sec Toutefois, il est significatif de noter que l'apparition du scorbut dans les deux cas ne montra pas de différence prononcée. Quand le lait liquide fut donné, le scorbut se développa en environ 3 semaines chez tous les animaux, sauf 2. Quoiqu'aucun signe extérieur de scorbut ne soit apparu chez ces animaux avant la $5^{\mathrm{e}}$ semaine, le déclin rapide après cette période et les résultats de l'autopsie prouvent son existence à une date antérieure. La durée moyenne du temps s'écoulant avant l'apparition du scorbut chez les animaux recevant le lait sec reconstitué fut d'environ 3 semaines, et tous les animaux montrent des symptômes nets avant la $5^{\mathrm{e}}$ semaine. 
TABLEAU V

RICHESSE COMPARÉE EN VITAMINE C DE 40 CC DE LAIT LIQUIDE ET DE LAIT SEC (DRVCO)

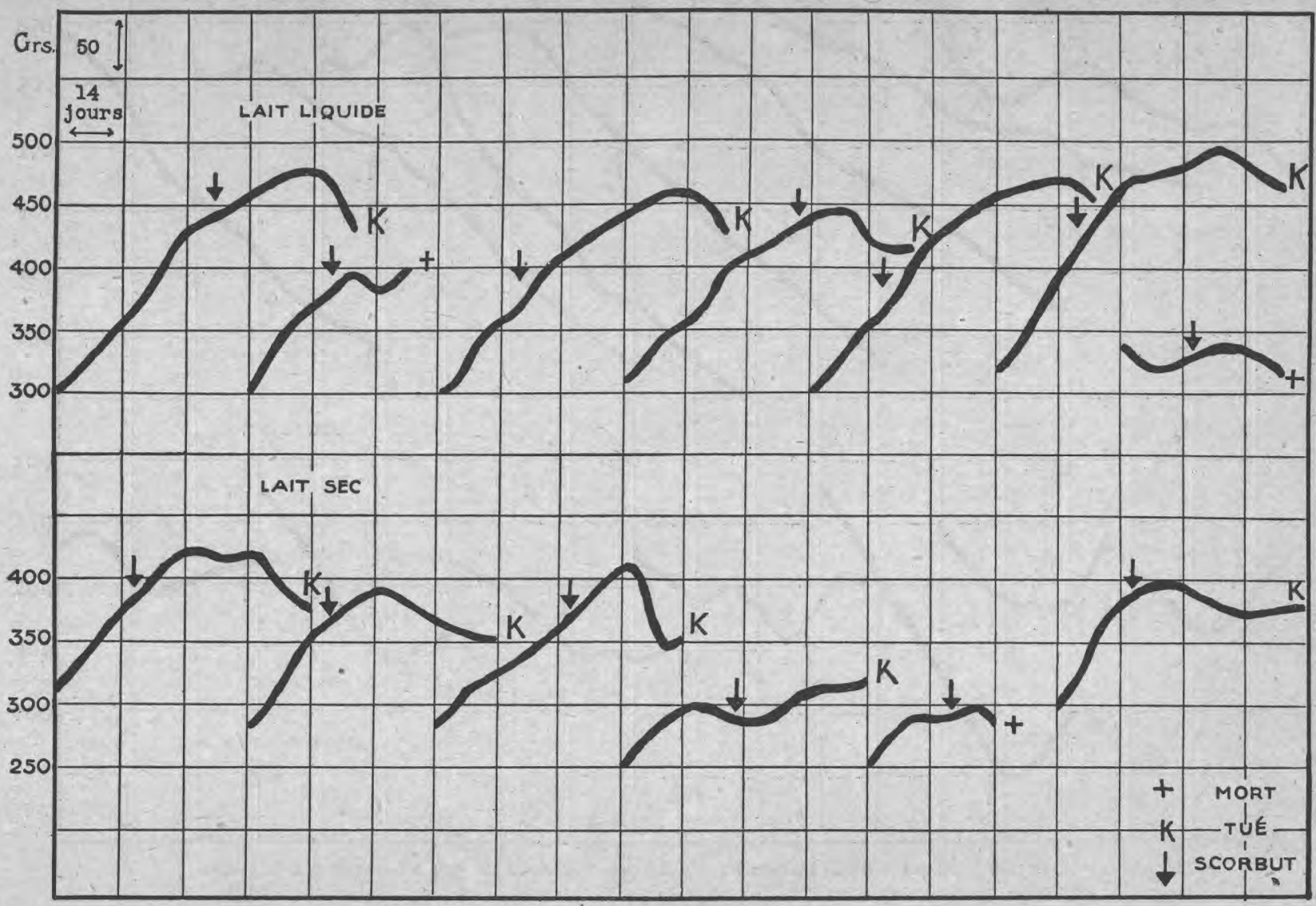




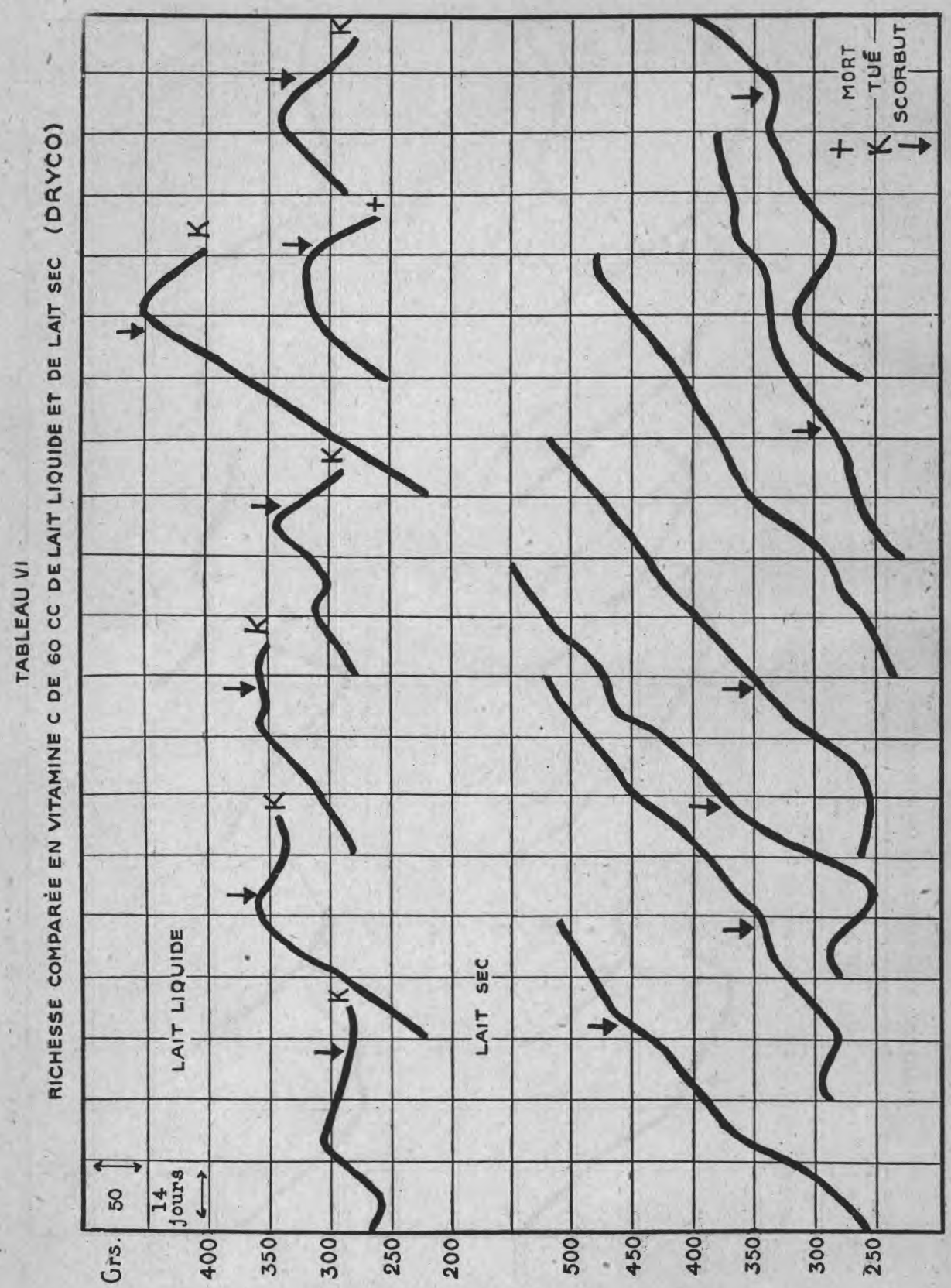


TABLEAU V1.

RICHESSE COMPARÉE EN VITAMINE C DE TO CC DE LAIT LIQUIDE ET DE LAIT SEC (DRYCO)

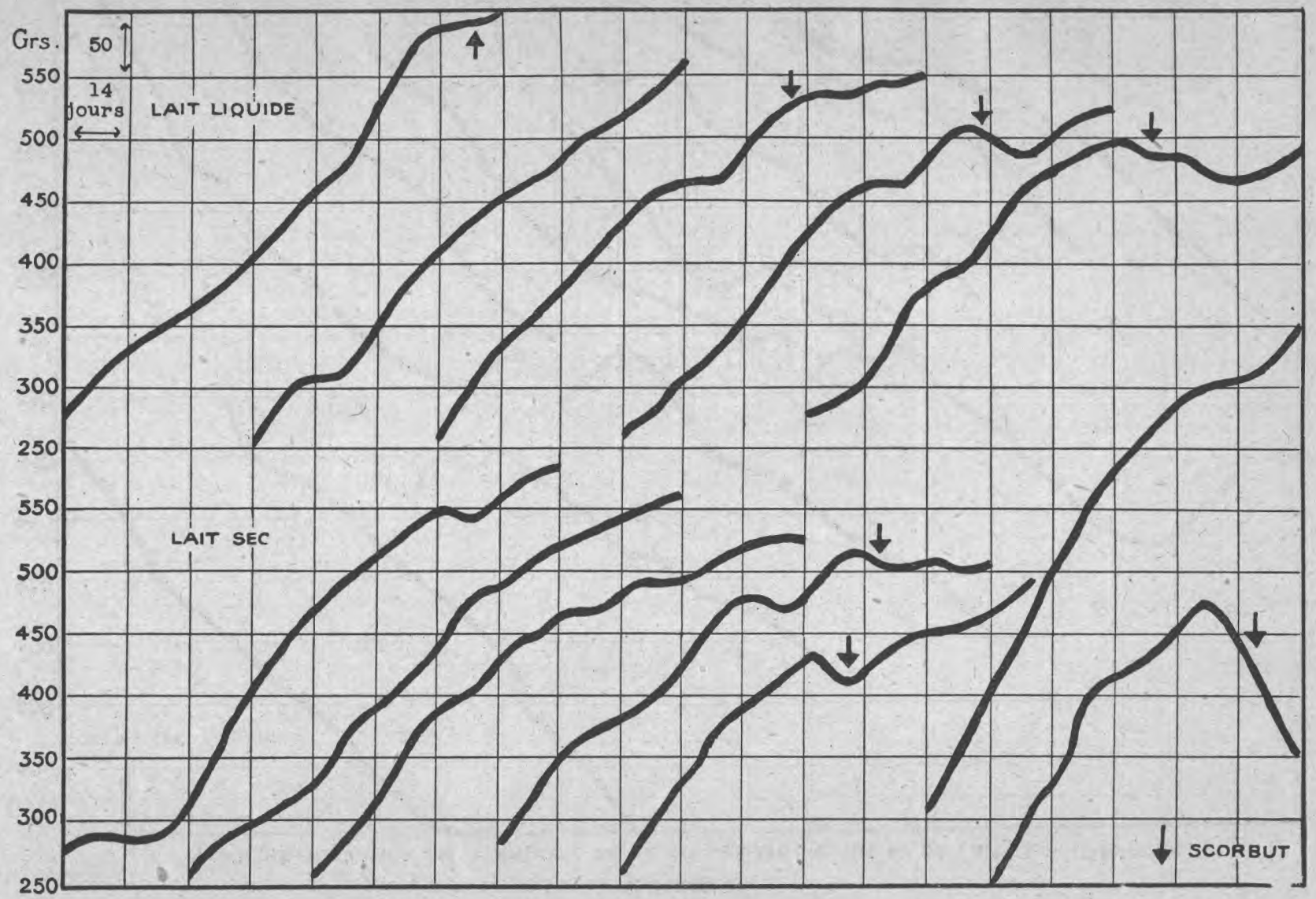




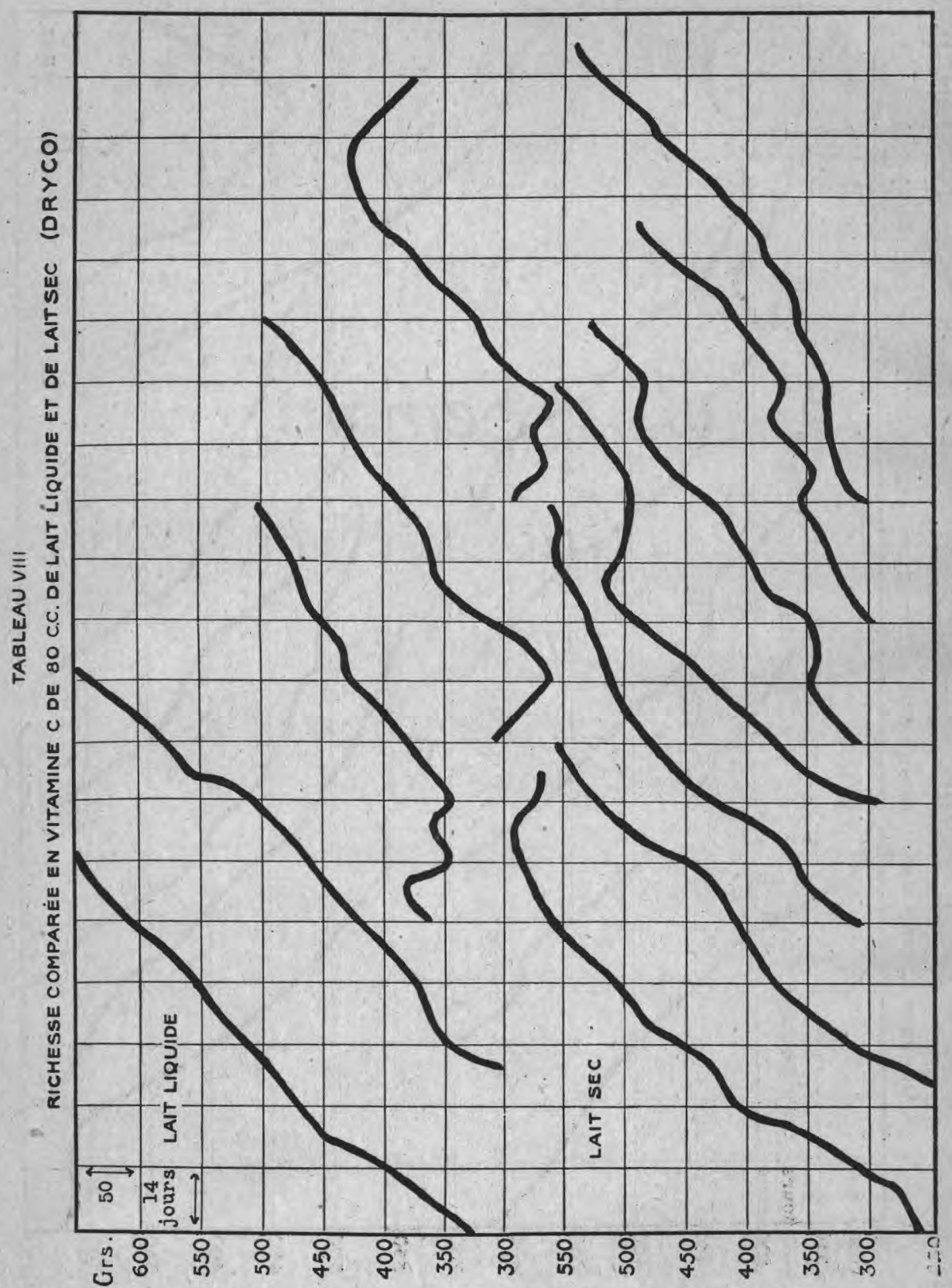


FOIRE

INTERNATIONALE

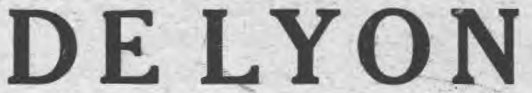

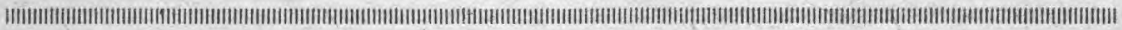

La Semaine

de la Machine Agricole aura lieu

du 12 au 20 Mars 1927

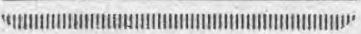

Elle se tiendra Cours de Verdun et groupera PLUS DE 300 ADHÉRENTS qui présenteront tout le Matériel agricole, vinicole horticole et de laiterie 


\section{Voici l'Unique Réfrigérant qui vous convient}

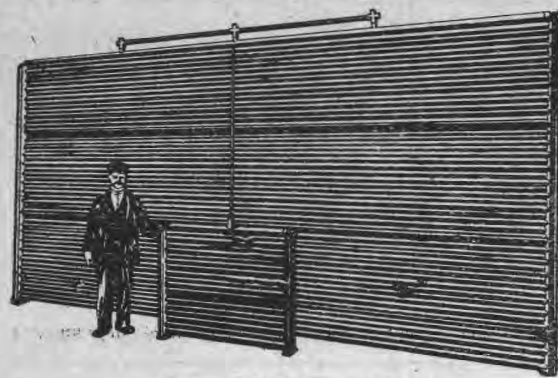

Deux Réfrigérants RINA

Lui scul vous donne le maximum de sarantie parce que coristruit par des Spécialistes en laiterie, dans leurs ateliers de Rueil (Seine-et-Oise).

$1 l$ refroidit à $1^{\circ}$ out $2^{\circ}$ d'écart.

Il consomme peu d'eau.

Sa fabrication est soignée en bronze et cuivre.

L'étamage complet est fäit d l'étain pur.

MAIS SURTOUT... il n'éclatè pas, il est éprouvé

à 10 kilos de pressian, extgez done cette garantie ailleurs.

Seuls Constructeurs en France des Réfrigérants à lait à détente directe de $\mathrm{CO}^{2}-\mathrm{NH}^{3}-\mathrm{SO}^{2}$.

Demandez nos Références et nos Prix défiant toute concurrence 10.000 litres de $90^{\circ}$ à $2^{\circ}$ à la Société Ampco, à Carentan. 1.000 litres de $85^{\circ}$ à $15^{\circ}$, à la Société Mondia.

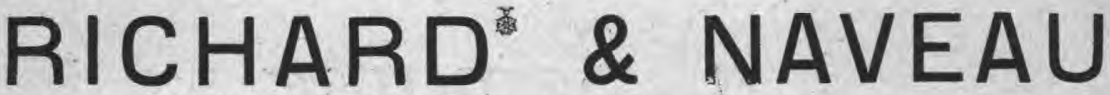

Construction française et Installation de Machines Frigorifiques et de Machines pour Laiteries

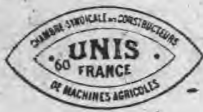

35, avenue de Paris - RUEIL (Seine-et-Oise)

Exposition de : Strasbourg : GRAND PRIX

R. C. Versailles 12.191

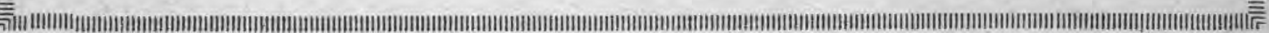

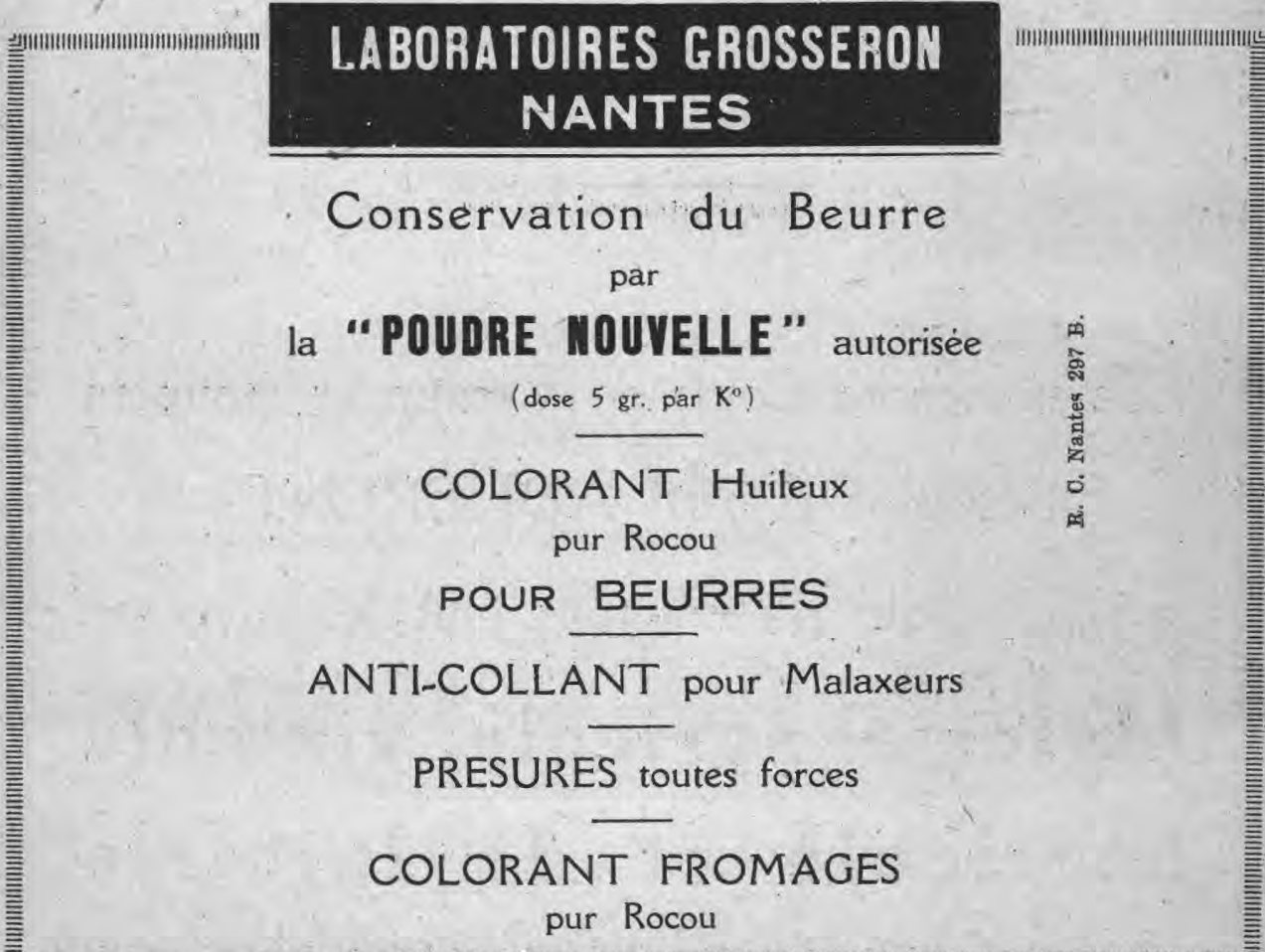

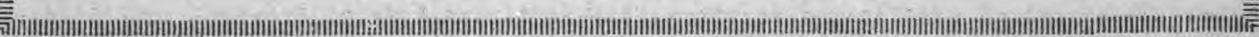




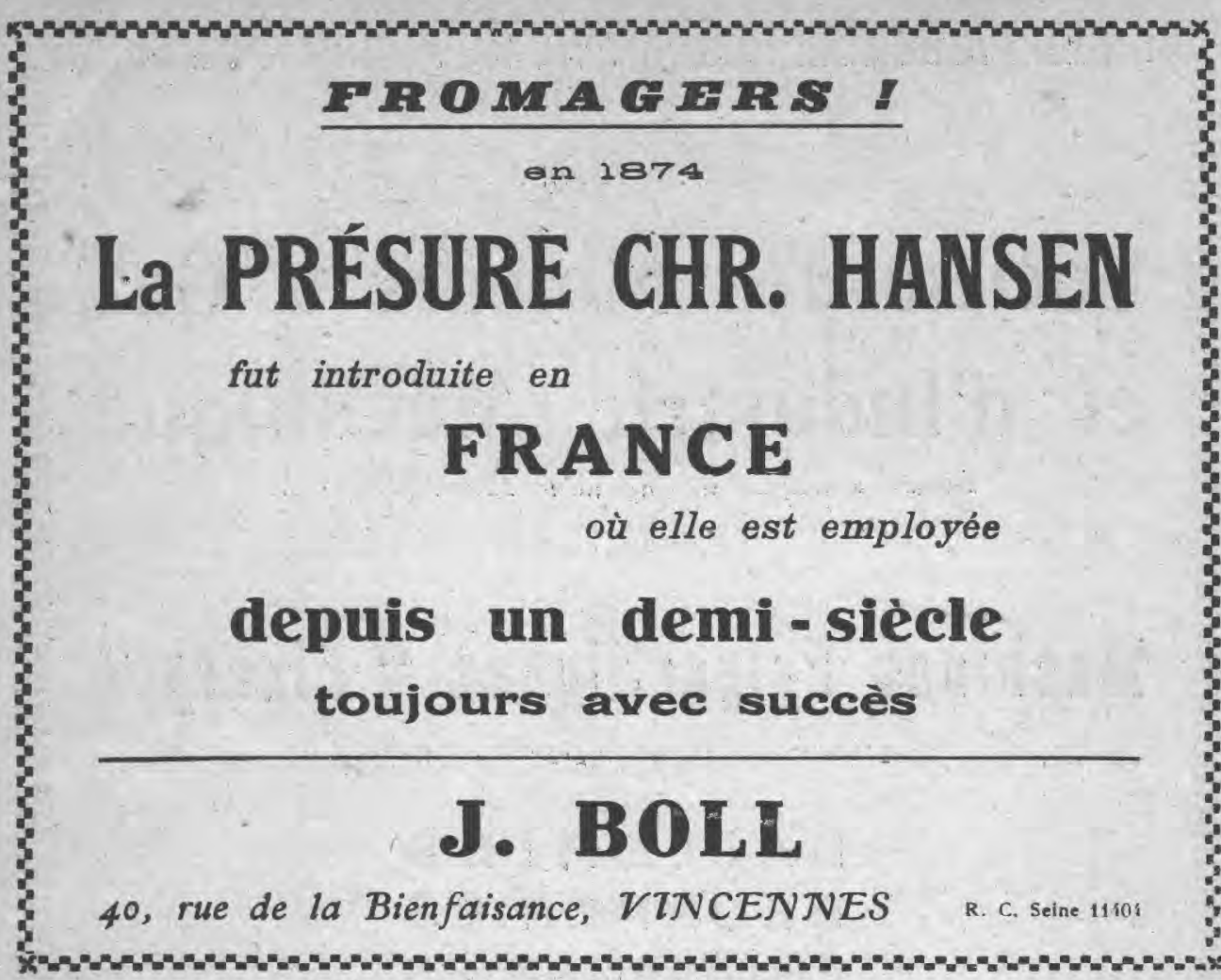

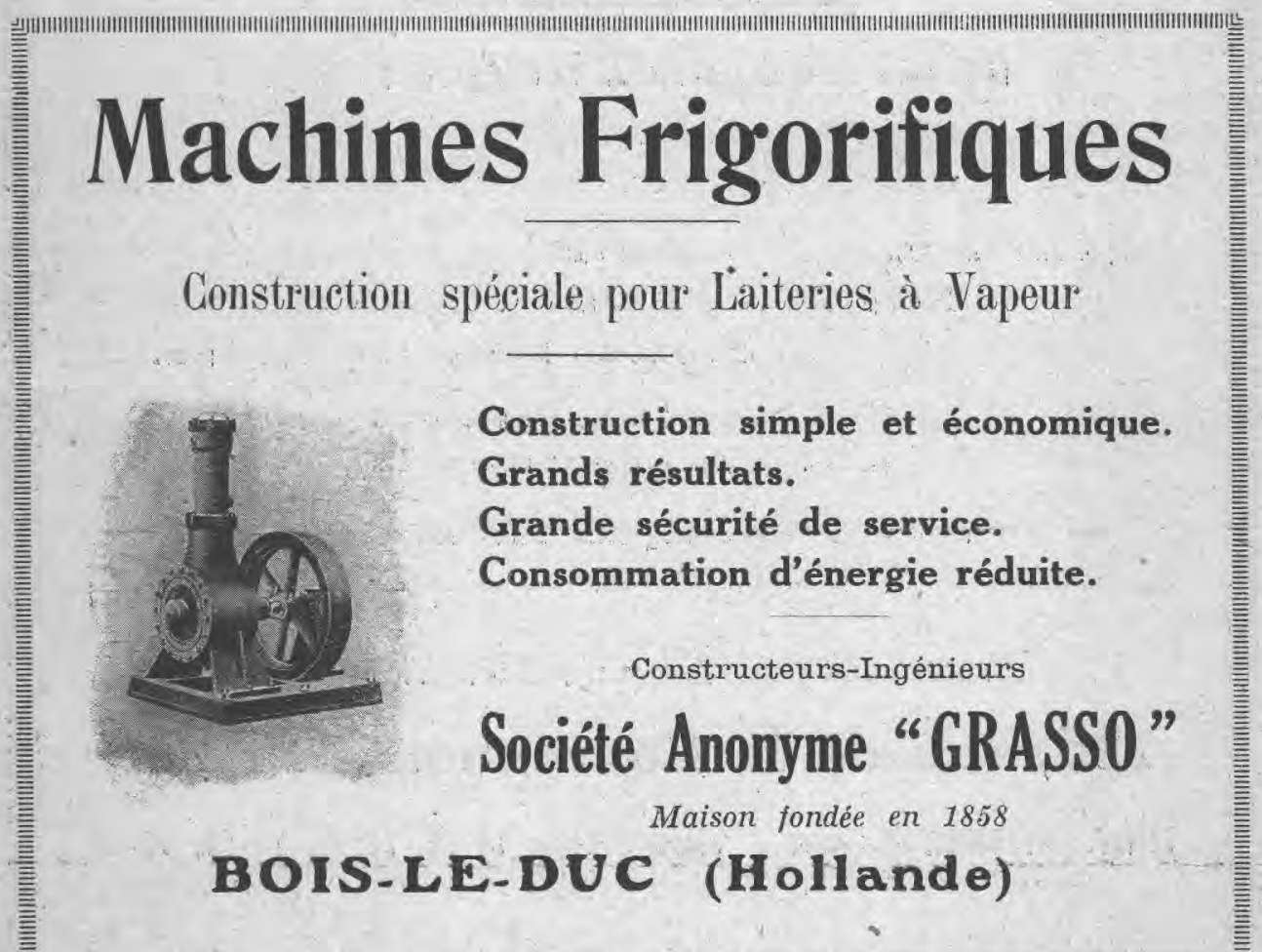


S.M.I.M.

\section{Société des Moteurs à Gaz}

et d'Industrie

Mécanique

Société Anonyme au Capital de 12.500.000 francs

Machines Frigorifiques " Fixary "

pour toutes applications

LAITERIES

BEURRERIES

FROMAGERIES

10 Installations à ROQUEFOR'T

Moteurs a Gaz. - Gazogènes

Moteurs a Pétrole, a Essence

Moteurs Ditesel et Semi-Diesel

POMPES CENTRIFUGES

pour toutes pressions et tous débits

Devis et Catalogues sur Demande

SIĖGE SOCIAL, BUREAUX ET ATELIERS:

135, rue de la Convention - PARIS

Télégr.: OTTOMOTEUR-PARIS - Téléph. : Ségur 74-13, 74-14, 74-15, 36-08 


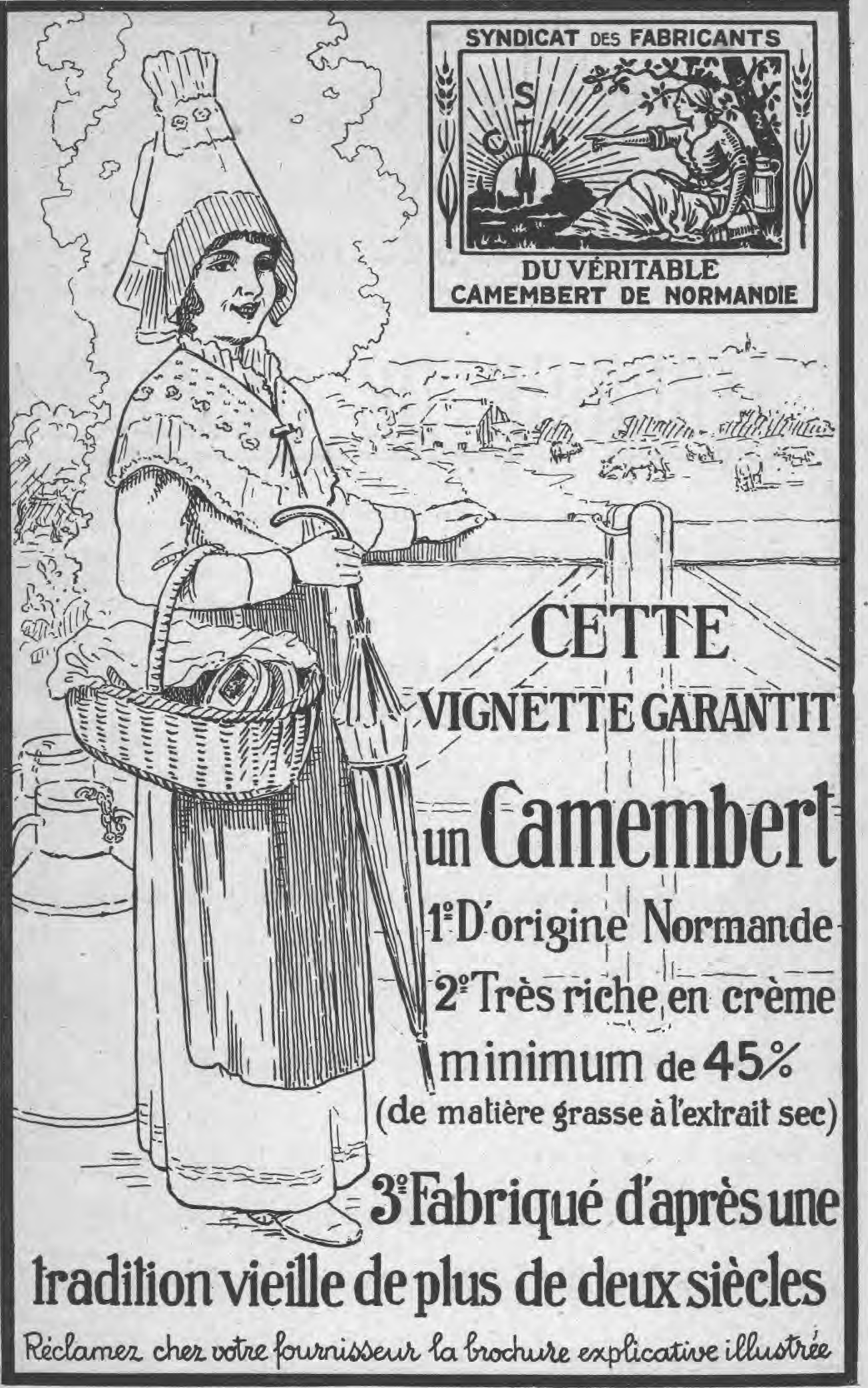




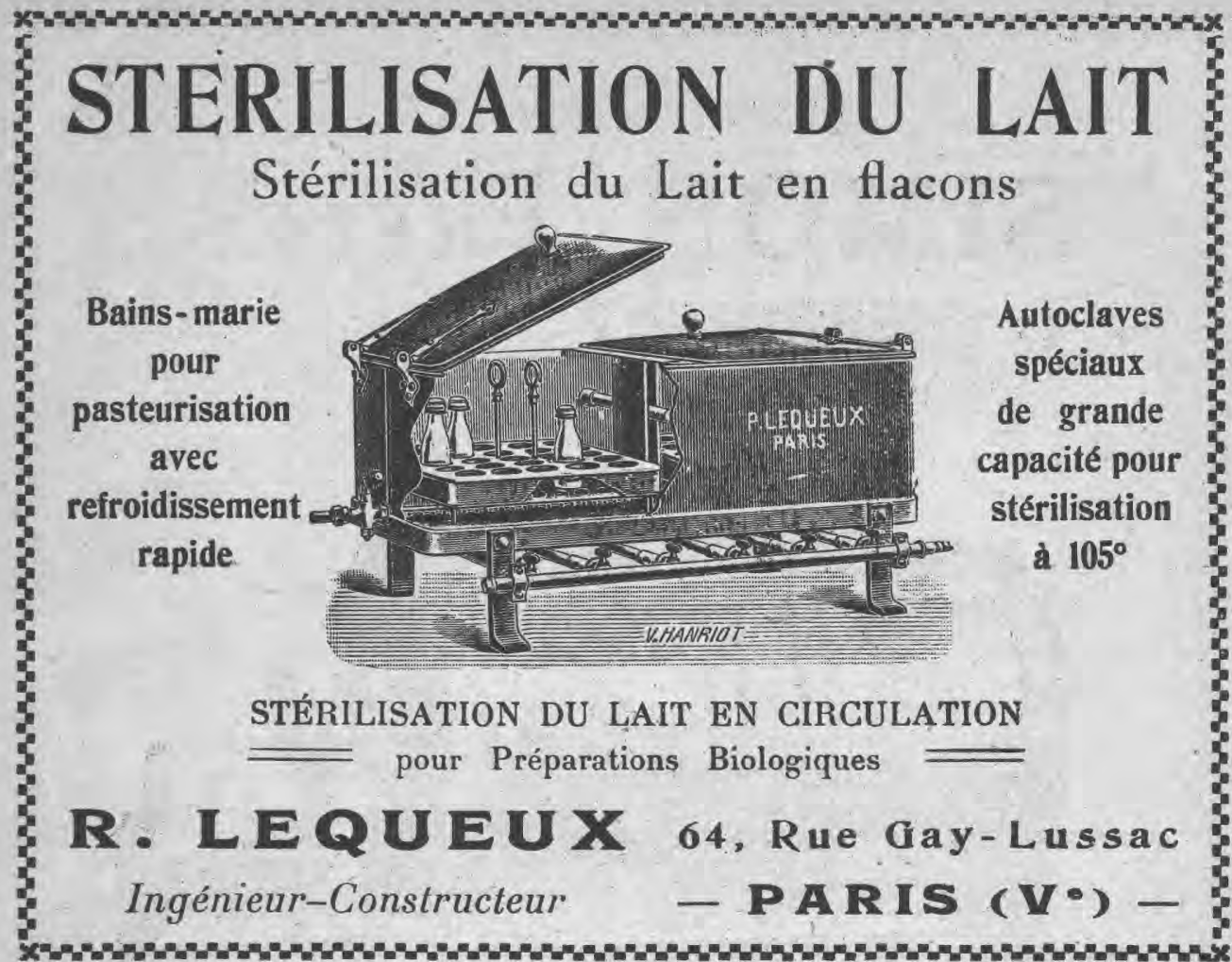

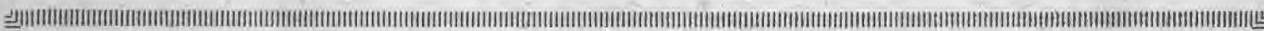

\section{Nous attirons votre attention}

sur le fait que notre Maison dite

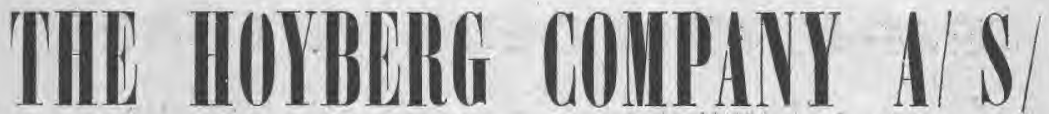

(DANSK MAELKETEKNISK LABORATORIUM A/S)

COPENHAGUE (DANEMARK)

est propriétaire des Brevets d'invention pris dans tous les pays sur le

\section{Procédé HOYBERG}

lequel sert à déterminer la quantité de matière grasse contenue dans le lait et la crème sans l'emploi des centrifuges et des acides.

Nous nous ferons un plaisir de répondre à toute demande de renseignements, et sommes prêts à adresser, avec des offres, la littérature, etc., concernant ledit procédé.

Nous sommes désireux d'entrer en relations, en tous pays, avec des naaisons de premier ordre dont les affaires sont orientées vers la branche laiterie. 
En ce qui concerne les animaux recevant $60 \mathrm{cc}$. (Tableau VI), tous, eurent le scorbut pendant la période expérimentale, sauf un qui ne montra aucun symptôme de la maladie, même à l'autopsie. La durée moyenne du temps s'écoulant avant que les animaux alimentés au lait liquide ne montrent des symptômes externes, fut de 5 semaines, tandis que la période moyenne pour l'apparition de symptômes similaires chez les animaux alimentés au lait sea reconstitué fut d'un peu plus de six semaines.

Les résultats indiqués dans le tableau VII pour des quantités de lait employées de $70 \mathrm{cc}$., ne montrent pas de différence impnrtante dans les propriétés antiscorbutiques entre le lait liquide et le lait sec. Quand le scorbut se développait, il se manifestait plus rapidement quand on employait du lajt sec, quoique la proportion des animaux prenant le scorbut fut plus grande dans le groupe recevant le lait liquide naturel. Nous eûmes de très grandes difficultés en raison de troubles digestifs avec le groupe d'animaux représenté dans le tableau VIII, animaux recevant $80 \mathrm{cc}$. de lait. Au début de la période expérimentale, une proportion considérable de la ration journalière étaitadministrée à la pipette et, sans aucun doute, eette méthode d'alimentation forcée aggrava encore le mal: Toutefois, en ce qui eoncerne la manifestation des symptômes du scorbut, il y a une uniformité nette dans le groupe entier. Aucun des animaux recevant $80 \mathrm{cc}$, soit de lait liquide, soit de lait sec reconstitué, n'eut le scorbut.

Les résultats de ces essais comparatifs avec du lait liquide et du lait sec fraîchement obtenu du même lait confirment les observations d'autres expérimentateurs prouvant qu'il y a peu ou pas de détérioration du facteur antiscorbutique dans le lait desséché par le procédé des doubles cylindres Just.

\section{Conclusions}

Les résultats des essais d'alimentation comparative montrent qu'il n'y a pas de détérioration mesurable des vitamines " $A$ ", " $B$ " ou " $C$ " pendant la dessic ation du lait frais par le procédé Just. Les détériorations ou diminutions de ces vitamines qui peuvent être remarquées dans des échantillons isolés de lait desséché par ce procédé doivent done être attribuées à d'autres facteurs que le procédé de dessiccation lui-même.

\section{BIBLIOGRAPHIE}

[1] Steeniock, H. et Nerson E.M. : Jour. Biol. Chem., Vol, LVI, 355, 1923.

[2] Steenbock H., Nelson M.T, et Black A. : Jour. Biol. Chem., Vol. LXII, $275,1924$.

[3] Streenbook H., Seli M.T. et Neison E.M. : Jour. Biol. Chem., Vol. LV, 399, 1923. 
[4] Stennbock H., Sent M. T. et Jones J. H.: Jour. Biol. Chem., Vol. LV, 4111923.

[5] Hess A.F. et UNGer L.J.: Jour. Biol. Chem., Vol. XXXVIII, 293, 1919.

[6] Hart E.B., Steenbock H. et Elurs N.R.: Jour. Biol. Chem., Vol. XLVI, $309,1921$.

[7] JoHnson J.M. et Hooper C.W.: Unites States Public Health Reports, Vol. XXXVII, 989, 1922.

[8] Jephcotт H. et Bacharach A.F. : Biochem. Jour., Vol. XV, 129, 1921.

]9] Supplee G.C. et Dow O.D.: American Journal of Diseases of Children Vol. XXXI, 41, 1926.

\section{RECHERCHES SUR LA QUALITE DU LAIT A MONTPELLIER}

Par F. CARRIEU,

Professeur Agrégé à la Faculté de Montpellier, Chế des Travaux d'Hygiène.

La qualité du lait, aliment de première nésessité pour l'enfant et le malude, a toujours interessé le médecin et l'hygiéniste. Dès 1906, on s'était préoccupé de la provenance ainsi que de la valeur alimentaire du lait consommé à Montpellier et l'on trouvera la réponse à ces deux questions dans le travail très complat de MM. BerTin-Sans et Ros [1]. En s'y reportant, on sera désagréablement surpris de voir qu'à cette époque 6 échantillons seulement sur 100 étaient irréprochables, 94 étaient anormaux (10 à 11 sont passablas, 17 à 18 sont mauvais, 66 à 67 "présentent des anomalies telles que l'on n'a plus en réalité affaire à du lait, mais plutôt à du pseudo-lait "). Il est bon d'ajouter que certains Iaitiers vendaient $0 \mathrm{fr}$. 40 le litre (et non $0 \mathrm{fr}, 30$ ) du lait qu'ils garantissaient exempts d'eau de mouillage. Or si, d'une façon générale, ce lait était bien supárieur à l'autre, certains de ces échantillons cependant étaient tout à fait défectueux, quelques-uns même inférieurs au lait à $0 \mathrm{fr}$. 30 , alors qu'ils provenaient pourtant de la même étable !

Devant cet état de choses, le Comité de l'Hérault de l'Alliance d'Hygiène sociale créa aussitôt (1907), une "Commission du lait de la Ligue contre la mortalité infantile \%. Cette Commission fut composée de MM. Bernis, inspecteur des Enfants assistés, H. BertinSans, H. Imbert et M. Moye, professeurs à l'Université, Belugou, BLaufus et GaUjoux, docteurs en médecine. Elle travailla activement jusqu'en 1914 et, chaque année, on constata une amélioration nouvelle. On obtint, en outre, la création d'un syndicat des laitiers qui acceptaient la surveillance de leurs produits et se soumettaient aux jugements de la Commission. Malheureusement, tous les laitiers de la ville ou des environs ne firent pas partie de ce syndicat et il est juste de noter que e'était parmi ces dissidents que les condamnations judiciaires furent, et de beaucoup, les plus nombreuses et les plus importantes. 